\title{
WHOLE-BODY-VIBRATION-INDUCED INCREASE IN Leg Muscle ACtivity During Different SQUAT EXERCISES
}

\author{
Machteld Roelants, ${ }^{1}$ Sabine M.P. Verschueren, ${ }^{2}$ Christophe Delecluse, ${ }^{1}$ Oron Levin, ${ }^{2}$ \\ AND VALÈRE STIJNEN ${ }^{1}$
}

\author{
${ }^{1}$ Exercise Physiology and Biomechanics Laboratory and ${ }^{2}$ Laboratory of Motor Control, Faculty of Physical \\ Education and Physiotherapy, Department of Kinesiology, Katholieke Universiteit Leuven, Leuven, Belgium.
}

\begin{abstract}
Roelants, M., S.M.P. Verschueren, C. Delecluse, O. Levin, and V. Stijnen. Whole-body-vibration-induced increase in leg muscle activity during different squat exercises. J. Strength Cond. Res. 20(1):124-129. 2006. - This study analyzed leg muscle activity during whole-body vibration (WBV) training. Subjects performed standard unloaded isometric exercises on a vibrating platform (Power Plate): high squat (HS), low squat (LS), and 1-legged squat (OL). Muscle activity of the rectus femoris, vastus lateralis, vastus medialis, and gastrocnemius was recorded in 15 men (age $21.2 \pm 0.8$ years) through use of surface electromyography (EMG). The exercises were performed in 2 conditions: with WBV and without (control [CO]) a vibratory stimulus of $35 \mathrm{~Hz}$. Muscle activation during WBV was compared with $\mathrm{CO}$ and with muscle activation during isolated maximal voluntary contractions (MVCs). Whole-body vibration resulted in a significantly higher $(p<0.05)$ EMG root-mean-square compared with $\mathrm{CO}$ in all muscle groups and all exercises (between +39.9 $\pm 17.5 \%$ and $+360.6 \pm 57.5 \%)$. The increase in muscle activity caused by WBV was significantly higher $(p<0.05)$ in OL compared with HS and LS. In conclusion, WBV resulted in an increased activation of the leg muscles. During WBV, leg muscle activity varied between 12.6 and $82.4 \%$ of MVC values.
\end{abstract}

KEY WoRDS. surface electromyography, vibration training, tonic vibration reflex, muscle strength

\section{INTRODUCTION}

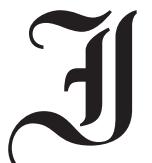

$\mathrm{t}$ has been shown that mechanical vibrations applied to the muscle or tendon stimulate sensory receptors, mainly length-detecting muscle spindles $(12,14)$. The activation of these muscle spindles facilitates the activation of alpha-motoneurons, leading to reflex muscle contractions (tonic vibration reflex) $(12,14)$. This response is mediated by monosynaptic and polysynaptic pathways and results in increased motor unit activation $(6,15)$. Several studies showed that whole-body vibration (WBV) training, in which subjects perform unloaded exercises on a vibrating platform, resulted in improved muscle strength or muscle performance $(3,11,19,20,24,25)$. Some other studies reported no changes in muscle strength after WBV training $(9,10)$.

Although WBV has been promoted as an alternative strength-training method in previously untrained subjects $(7,11,19,20)$, there still exists a lack of knowledge about the responsiveness of the neuromuscular system during vibration. So far, only a couple of studies have reported basic electromyography (EMG) recordings during vibration. One study showed that vibration applied on the arm during maximal dynamic elbow flexion almost doubled the EMG root-mean-square (rms) values in the biceps brachii muscle compared with the same exercise without vibration (2). Some studies reported an increase in leg muscle activity while subjects stood on a WBV platform $(8,16)$. However, the responsiveness of different muscles in different exercises during WBV has not been studied. First, it is not clear whether specific exercises performed on the WBV platform are more effective to evoke additional muscle activity than are other exercises. Second, it is not clear how much muscles are activated during WBV compared with an isolated maximal voluntary contraction of that specific muscle. Therefore, this study aims to investigate the magnitude of WBV-induced increase in the activity of different leg muscles in subjects performing 3 standard unloaded isometric exercises: high squat (HS), low squat (LS), and 1-legged squat (OL).

First, it is hypothesized that in all exercises the activity of the leg muscles will be higher when performed on a vibrating platform. Second, it is hypothesized that the magnitude of the WBV-induced increase in leg muscle activity will depend on the type of squat exercise performed on the vibrating platform. The sensitivity of the muscle spindles, which are assumed to be the main contributors to the reflex muscle activation $(12,14)$, is higher in stretched muscles and preactivated muscles $(4,5)$. Therefore, it is expected that an exercise inducing a high stretch or a high preactivation of the receptor-bearing muscle will result in a larger increase in muscle activity during WBV as compared with an exercise with a shorter or more relaxed state of that muscle. Finally, it is questioned to what extent muscle activation on a vibrating platform approaches muscle activation during an isolated maximal voluntary contraction (MVC) of that specific muscle.

A better insight in muscle activation while performing standard exercises on a WBV platform is undoubtedly helpful to determine the potential of WBV programs in revalidation and training.

\section{Methods}

\section{Experimental Approach to the Problem}

To test both hypotheses put forward in the introduction, this study analyzed the leg muscle activity during WBV training while subjects performed standard unloaded isometric exercises: HS, LS, and OL. Muscle activity of the rectus femoris, vastus lateralis, vastus medialis, and gastrocnemius was recorded through use of surface EMG in the different exercises with WBV and without (control 
[CO]) a vibratory stimulus of $35 \mathrm{~Hz}$ (Power Plate). For comparison, muscle activation during isolated maximal voluntary contractions was recorded.

\section{Subjects}

Fifteen male physical education students (age $21.2 \pm 0.8$ years, height $179.1 \pm 5.9 \mathrm{~cm}$, weight $76.7 \pm 7.6 \mathrm{~kg}$ ) volunteered to participate in this study. Reasons for exclusion were acute hernia, any history of severe musculoskeletal problems, diabetes, or epilepsy. All subjects gave written informed consent to participate. The study was approved by the University's Human Ethics Committee according to the declaration of Helsinki.

\section{EMG Analysis}

The surface EMG signals (Noraxon Myosystem 2000, Scottsdale, AZ) from the rectus femoris, vastus lateralis, vastus medialis, and gastrocnemius (medial) muscle of the dominant leg were recorded bipolarly by disposable 20-mm disc electrodes (Blue Sensor $\mathrm{Ag} / \mathrm{AgCl}$ ). The electrodes were fixed lengthwise over the middle of the muscle belly with an interelectrode (center-to-center) distance of $25 \mathrm{~mm}$. The reference electrode was attached to the right upper arm. The preamplified EMG signals were amplified $(\times 1,000)$, bandpass filtered $(15 \mathrm{~Hz}-10 \mathrm{kHz})$, and sampled at 2,000 Hz (CED Power 1401, Cambridge Electronic Devices, Cambridge, UK) for off-line analysis. Electromyography cables were fastened to prevent the cables from swinging and from movement artifact.

\section{Treatment Protocol}

After positioning of the electrodes, the experimental session started with a standardized warm-up consisting of 5 minutes of cycling on an ergometer without resistance. Before the WBV test protocol was started, muscle activity was recorded during performance of isolated MVCs. The MVCs were performed isometrically. Those involving the rectus femoris, vastus lateralis, and vastus medialis muscle were recorded during an isolated leg extension at knee angles of $90^{\circ}$ and $125^{\circ}$ with the subjects supine on a therapy table with knees hanging of the table. Maximum voluntary contraction involving the gastrocnemius muscle was determined by means of plantar flexion of the foot with subjects supine and with an ankle angle of $90^{\circ}$.

Muscle activity was recorded during 3 standard unloaded isometric exercises with and without WBV: HS (knee angle $125^{\circ}$, hip angle $140^{\circ}$ ), LS (knee and hip angles $90^{\circ}$ ), and $\mathrm{OL}$ (knee angle $125^{\circ}$, hip angle $140^{\circ}$ ). Posture was strictly controlled during all exercises by standardizing knee and hip joint angles. In addition, a straight back was required during all exercises. Each subject familiarized himself with the conditions of all exercises and with the WBV stimulus before testing.

Electromyography recordings started when the subjects stood in the correct posture (HS, LS, or OL) on the vibration platform (Power Plate). In the CO condition, muscle activity was recorded for 30 seconds while subjects stood on the platform without WBV. In the WBV condition, muscle activity was recorded during 5 seconds before WBV. For the next 20 seconds, vibration was applied at a frequency of $35 \mathrm{~Hz}$ and amplitude of $2.5 \mathrm{~mm}$. When the 20 seconds of WBV were finished, the subjects were instructed not to alter their posture for 5 more seconds. Hereafter, the EMG registration stopped.

Four sets of each type of exercise were performed, and the 3 exercises were applied in random order in both the $\mathrm{CO}$ the WBV conditions. Between each exercise and between each set, subjects sat down on a chair for 1 minute to unload the muscle spindles and to avoid aftereffects of vibration.

\section{Data Analysis}

The amplified raw EMG signal was converted to an average rms signal. The mean EMGrms value over a range of 20 seconds (range between 5 and 25 seconds of the registration) was computed for each exercise in each muscle group in the $\mathrm{CO}$ condition and the WBV condition. The mean EMGrms value of the 4 sets of each exercise was selected for statistical analyses. The effect of WBV on muscle activity is called vibration effect and is defined as the WBV-induced increase in EMGrms compared with the $\mathrm{CO}$ condition. Muscle activity during the $\mathrm{CO}$ and WBV conditions was also expressed relative to the measured MVC values (\%MVC): for HS and DL squat, quadriceps muscle activity (rectus femoris, vastus lateralis, and vastus medialis) was expressed relative to muscle activity during maximal leg extension at a knee angle of $125^{\circ}$ for LS, quadriceps muscle activity was expressed relative to muscle activity during maximal leg extension at a knee angle of $90^{\circ}$. Gastrocnemius muscle activity was expressed relative to muscle activity during maximal plantar flexion of the foot at an ankle angle of $90^{\circ}$. The data are reported as mean $\pm S E$.

\section{Statistical Analyses}

Statistical analysis was performed with an analysis of variance for repeated measures (2 [condition] $\times 3$ [type of exercise]). After an overall $F$ value was found to be significant, preplanned contrast analyses were performed to evaluate significant vibration effects in each exercise and significant differences in vibration effect among exercises. A Bonferroni correction was used to adjust the $p$ value in relation to the number of contrasts that were performed. All analyses were executed by the statistical package Statistica, version 6 (Statsoft, Inc., Tulsa, OK). Significance level was set at $p \leq 0.05$.

\section{RESULTS}

\section{Rectus Femoris Muscle}

As can be seen in Figure 1a and Table 1, representing the muscle activity of the rectus femoris during different exercises, EMGrms activity was always higher in the WBV condition compared with the $\mathrm{CO}$ condition without vibration. Statistical analyses confirmed these observations: a significant 'condition' effect was found $(F(2)=9.6, p<$ $0.001)$. Contrast analyses clarified a significant $(p<$ 0.001) WBV-induced increase in EMGrms activity in HS $(+115.1 \pm 16.3 \%), \mathrm{LS}(+49.1 \pm 6.7 \%)$, and OL $(+151.4 \pm$ $19.5 \%)$. In addition, differences in vibration effect among exercises were found as the 'condition $\times$ exercise' interaction effect was significant $(F(2)=11.2, p<0.001)$. The vibration effect in OL was significantly higher $(p<0.01)$ compared with HS and LS. The vibration effect in HS was not significantly different $(p>0.05)$ from LS.

\section{Vastus Medialis Muscle}

As can be seen in Figure $1 \mathrm{~b}$ and Table 1, representing the muscle activity of the vastus medialis during different exercises, EMGrms activity was higher in the WBV condi- 


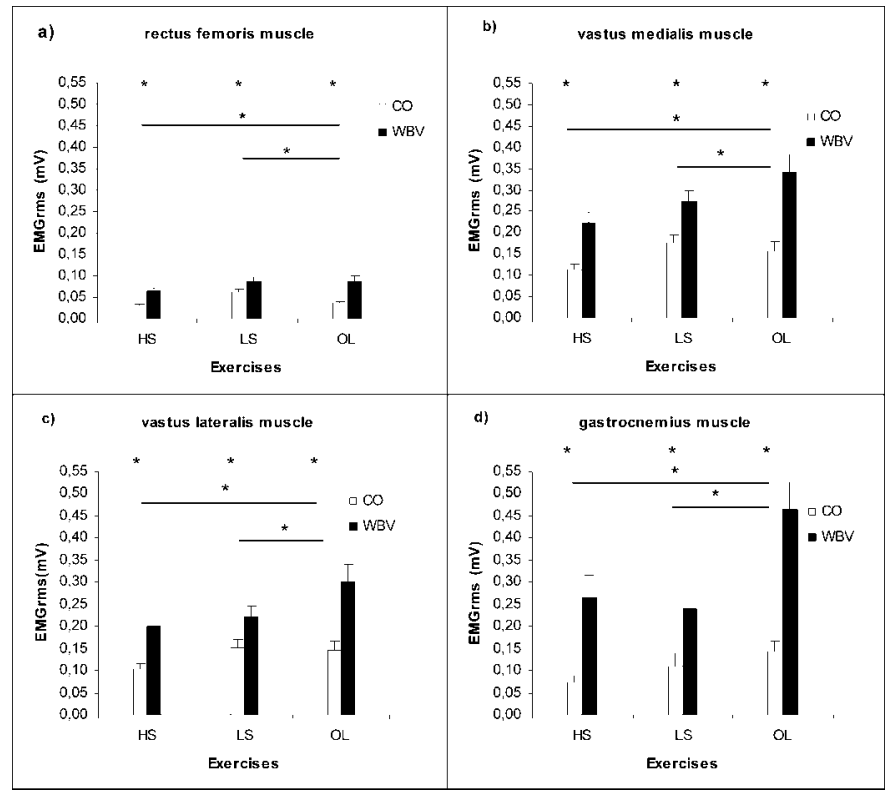

FIGURE 1. Electromyography root-mean-square $(\mathrm{mV})$ in high squat (HS), low squat (LS), and 1-legged squat (OL) in the rectus femoris muscle (a), vastus medialis muscle (b), vastus lateralis muscle (c), and gastrocnemius muscle (d) in the control (CO) and the whole body vibration (WBV) conditions. *Significant increase in muscle activity CO-WBV $(p<0.05)$. * Significant difference in vibration effect among exercises $(p$ $<0.05)$. Values are mean $\pm S E$.

tion compared with the $\mathrm{CO}$ condition without vibration $(F(2)=18.8, p<0.001)$. Contrast analyses clarified a significant $(p<0.001)$ WBV-induced increase in EMGrms activity in HS $(+102.0 \pm 14.4 \%), \mathrm{LS}(+59.0 \pm$ $7.4 \%)$, and OL $(+124.7 \pm 9.9 \%)$. In addition, significant differences in vibration effect among exercises were found $(F(2)=23.1, p<0.001)$. The vibration effect in OL was significantly higher $(p<0.001)$ compared with HS and LS. The vibration effect in HS was not significantly different $(p>0.05)$ from LS.

\section{Vastus Lateralis Muscle}

As can be seen in Figure 1c and Table 1, representing the muscle activity of the vastus lateralis during different exercises, EMGrms activity was higher in the WBV condition compared with the $\mathrm{CO}$ condition without vibration $(F(2)=8.9, p<0.001)$. Contrast analyses clarified a significant $(p<0.001)$ WBV-induced increase in EMGrms activity in $\mathrm{HS}(+92.5 \pm 14.8 \%), \mathrm{LS}(+51.7 \pm 7.8 \%)$, and OL $(+115.3 \pm 15.2 \%)$. In addition, significant differences in vibration effect among exercises were found $(F(2)=$ $19.5, p<0.001)$. The vibration effect in OL was significantly higher $(p<0.01)$ compared with HS and LS. The vibration effect in HS was not significantly different $(p>$ 0.05) from LS.

\section{Gastrocnemius Muscle}

As can be seen in Figure 1d and Table 1, representing the muscle activity of the gastrocnemius during different exercises, EMGrms activity was higher in the WBV condition compared with the $\mathrm{CO}$ condition without vibration $(F(2)=19.4, p<0.001)$. Contrast analyses clarified a significant $(p<0.001)$ WBV-induced increase in EMGrms activity in HS $(+301.3 \pm 48.8 \%), \mathrm{LS}(+134.1 \pm$

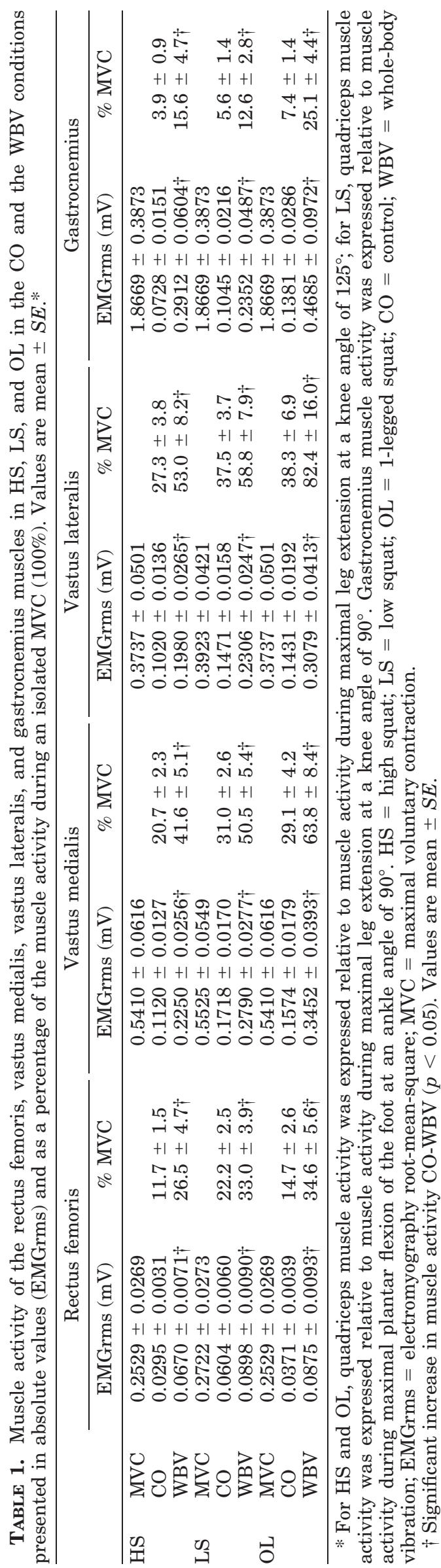


$20.6 \%)$, and OL (+360.6 $\pm 57.5 \%)$. In addition, significant differences in vibration effect among exercises were found $(F(2)=26.5, p<0.001)$. The vibration effect in OL was significantly higher $(p<0.01)$ compared with HS and LS. The vibration effect in HS was not significantly different $(p>0.05)$ from LS.

\section{Maximal Voluntary Contraction}

Table 1 represents the muscle activity of the different muscles in the $\mathrm{CO}$ condition and the WBV condition expressed as a percentage of the muscle activity during an isolated MVC. All muscles showed significant increases $(p>0.05)$ in muscle activity (\%MVC) due to WBV.

During WBV exercises, the muscle activity expressed as a percentage of MVC values (100\%) varied depending on the exercise: between 26.5 and $34.6 \%$ in the rectus femoris muscle, between 41.6 and $63.8 \%$ in the vastus medialis muscle, between 53.0 and $82.4 \%$ in the vastus lateralis muscle, and between 12.6 and $25.1 \%$ in the gastrocnemius muscle. During WBV, the EMG activity of the vastus medialis and vastus lateralis muscles in OL reached up to $63.8 \pm 8.4 \%$ and $82.4 \pm 16.0 \%$ of the MVC values, respectively.

\section{Discussion}

This is the first study that focuses on the WBV-induced increase in activity of the leg muscles during standard unloaded isometric exercises: HS, LS, and OL. The first hypothesis of this study was confirmed because the results showed that muscle activity (EMGrms) increased significantly during WBV at $35 \mathrm{~Hz}$ in all muscles and all exercises compared with the nonvibrating $\mathrm{CO}$ condition. In line with the second hypothesis, the magnitude of this increase in muscle activity caused by WBV (vibration effect) was different among exercises and among muscles, showing a variation between $+39.9 \pm 17.5 \%$ and +360.6 $\pm 57.5 \%$. The magnitude of the vibration effect was significantly higher in OL compared with HS and LS. During WBV, activation of the leg muscles varied between 12.6 and $82.4 \%$ of MVC values.

Cardinale and Lim (8) reported an increase of 34\% in vastus lateralis muscle EMGrms when subjects stood in an HS position on a platform vibrating at $30 \mathrm{~Hz}$ with an amplitude of $10 \mathrm{~mm}$. This increase is clearly lower compared with the WBV-induced increase in vastus lateralis muscle activity of $92.5 \pm 14.8 \%$ in HS in the present study. It is reasonable to accept that this difference in vibration effect may be caused by differences in study design: in vibration frequency ( $30 \mathrm{~Hz}$ vs. $35 \mathrm{~Hz}$ ), in vibration amplitude (10 $\mathrm{mm}$ vs $2.5 \mathrm{~mm}$ ), and in HS knee joint angle $\left(100^{\circ}\right.$ vs. $\left.125^{\circ}\right)$. Nevertheless, in both studies a clear increase in muscle activity (EMGrms) during WBV has been shown, and this may be explained through muscle spindle-induced reflexive recruitment of previously inactive motor units and by synchronization among active motor units $(13,15)$.

In line with the second hypothesis, the magnitude of WBV-induced increase in muscle activity was different among exercises. These findings are in accordance to the EMG responses of the arm and shoulder muscles to vibrations during different postures of drilling (21). Muscles with an increased muscle length or increased degree of preactivation seemed to be most affected by vibration (21). Two main factors were selected as potential media- tors of the vibration effect: (a) the muscle length and (b) the muscle preactivation $(13,16)$.

The vibration effect was expected to be larger in exercises in which the muscles are more stretched because of an increased sensitivity of muscle spindles during stretch $(4,17)$. It is clear that the lengths of the monoarticular vastus lateralis and vastus medialis muscles are higher when the knees are more bent. However, in these muscles the vibration effect was not significantly larger during LS compared with HS. Unfortunately, changing the posture from HS to LS changes more parameters than only the muscle length (e.g., muscle preactivation), which might mask the length effect. Further studies comparing exercises with more-extreme and better-controlled differences in muscle length would probably reveal the impact of muscle length on the magnitude of the vibration effect. However, this study focused on the vibration effect in traditional WBV exercises.

A second factor that may play a role in the vibration sensitivity of a muscle is the degree of 'initial' muscle activity during the particular exercise in the $\mathrm{CO}$ condition. One study showed that the tonic vibration reflex increased with the initial contraction level of the muscle (18). It was expected that the vibration effect would be larger in exercises in which the receptor-bearing muscle is more preactivated before the start of vibration, as muscle spindles are more sensitive to vibration during a voluntary contraction compared with a relaxed muscle (5, 22 ). In the present study, the increase in muscle activity caused by WBV was significantly higher in OL compared with HS and LS. When supporting the total body weight on $1 \mathrm{leg}$ (in OL) instead of 2 legs (in HS), there is a small increase $(p>0.05)$ in muscle activity. Even a small increase in preactivation may lead to increased musclespindle sensitivity because of alpha-gamma coactivation (5) and might therefore explain the larger vibration effect in OL compared with HS. However, the higher preactivation in LS compared with OL does not result in a larger vibration effect of the former. Thus, it is clear that the level of preactivation does not explain the whole picture.

In this respect, it must be stated that the hypotheses regarding the mechanisms of WBV on muscle activation, such as the influence of the muscle length and muscle preactivation $(4,5)$, are mainly based on findings of isolated muscle vibration $(2,3,7,11,25)$. It remains unknown to what extent these findings can be linked to WBV $(9,10)$.

In the present study, the vibration effect was clearly dependent on the distance between the muscle and the vibration platform. For example, in HS (Figure 1) the relative WBV-induced increase in activity of the gastrocnemius muscle was clearly higher $(+301.3 \pm 48.8 \%)$ compared with the thigh muscles farther from the vibration platform: rectus femoris muscle $(+115.1 \pm 16.3 \%)$, vastus medialis muscle $(+102.0 \pm 14.4 \%)$, and vastus lateralis muscle $(+92.5 \pm 14.8 \%)$. It is obvious that in muscles closer to the WBV platform the vibration stimulus is less damped because of muscle and segment stiffness (16).

The findings of this study undoubtedly showed a substantial increase in muscle activation during WBV vs. CO conditions. The OL resulted in the highest vibration effect. When determining the potential role of WBV exercises as a strength-training stimulus, both the magnitude of the vibration effect and the total degree of muscle activation are important. During WBV, muscle activity of 
the vastus medialis and vastus lateralis muscles in $\mathrm{OL}$ reached up to $63.8 \pm 8.4 \%$ and $82.4 \pm 16.0 \%$ of the MVC values. In HS and LS, activity of the vastus lateralis and vastus medialis muscles during WBV was between 41.6 $\pm 5.1 \%$ and $58.8 \pm 7.9 \%$ of the MVC values. Muscle activity of the rectus femoris muscle during WBV reached less than $50 \%$ of maximum activation in all exercises (Table 1). It can be questioned whether these low-to-moderate muscle activity levels are sufficient as strength-training stimuli, especially when we consider the fact that muscle activation during WBV in this study was expressed as a percentage of the MVC activation recorded during an isolated muscle contraction. A recent study showed significantly higher quadriceps muscle activity during a maximal isometric squat compared with a more isolated activation of the quadriceps muscle by means of a maximal leg extension (1). If the muscle activity during WBV in current study had been expressed relative to maximal squat values, muscle activation would probably have been estimated lower. Notwithstanding a moderateto-low degree of muscle activation during WBV, several studies clearly showed that WBV training resulted in improved knee-extensor strength and jump performance, comparable to the gains recorded after resistance training at moderate intensity $(11,19,20)$. A placebo-controlled study using the same vibration parameters $(35 \mathrm{~Hz}$ and $2.5 \mathrm{~mm}$ ) as in this study reported that the strength increases after 12 weeks of WBV training in untrained women $(20 \times 1$-minute vibration exposure per session $)$ were clearly associated with the WBV-induced increase in muscle activity and were not attributable to the unloaded exercise program performed on the platform (11). Another study using a different WBV program $(30 \mathrm{~Hz}, 8$ $\mathrm{mm}, 8 \times 1$-minute vibration exposure per session) reported no improvement in muscle performance after 11 weeks of WBV training (10). Several authors suggested that strength gain after WBV training may be related to neural adaptations in absence of morphological adaptations of the muscle $(3,7,11,20)$. Neural adaptation can explain why several studies found improved muscle performance (23) after WBV training even when muscle activation during WBV in certain muscles was rather low to moderate.

In conclusion, the present study analyzed the WBVinduced increase in activity of the leg muscles during unloaded isometric standard exercises: HS, LS, and OL. The results clearly showed in all exercises a significant increase in activity (EMGrms) of the tested leg muscles during WBV at $35 \mathrm{~Hz}$ compared with the $\mathrm{CO}$ condition. The OL resulted in the largest WBV-induced increase in muscle activity compared with HS and LS. During WBV, activation of the leg muscles varied between 12.6 and $82.4 \%$ of MVC values.

\section{Practical Applications}

This study analyzed leg muscle activity during WBV. Subjects performed the most commonly used isometric exercises on a vibrating platform $(35 \mathrm{~Hz}$ and $2.5 \mathrm{~mm})$ : $\mathrm{HS}$, LS, and OL. Coaches and physiotherapists should know that in all 3 exercises WBV elicits a significant increase in leg muscle activity compared with the same exercises without WBV. This clear increase in muscle activity caused by WBV (vibration effect) is different among exercises and among muscles, showing a variation between $+39.9 \pm 17.5 \%$ and $+360.6 \pm 57.5 \%$. The OL exercise is most effective to evoke additional muscle activity caused by WBV. Leg muscles located closer to the vibration platform (gastrocnemius muscle vs. rectus femoris muscle) show a higher vibration effect.

During WBV, activation of the leg muscles varied between 12.6 and $82.4 \%$ of values recorded during an isolated MVC of that muscle. Although leg muscle activation during WBV reached up to $82.4 \%$ of MVC values in OL exercises, muscle activation in LS and HS exercises was low to moderate. This means that the musculoskeletal impact of WBV in certain muscle groups during these squat exercises is rather limited. However, WBV may have a high impact on the neuromuscular system $(3,7,11,19)$. This probably explains why several previous studies reported gains in muscle strength or performance after WBV in untrained subjects $(11,19,20,25)$.

This study gave a better insight in the responsiveness of the neuromuscular system during WBV. This information will help professionals involved in the health, fitness, and therapeutic sectors to determine the potential of WBV programs in revalidation and training.

\section{REFERENCES}

1. Behm, D.G., J.E. Power, AND E.J. Drinkwater. Muscle activation is enhanced with multi- and uni-articular bilateral versus unilateral contractions. Can. J. Appl. Physiol. 28:38-52. 2003.

2. Bosco, C., M. Cardinale, and O. Tsarpela. Influence of vibration on mechanical power and electromyogram activity in human arm flexor muscles. Eur. J. Appl. Physiol. 79:306-311. 1999.

3. Bosco, C., M. Iacovelli, M.O. Tsarpela, M. Cardinale, M. Bonifazi, J. Tihanyi, M. Viru, A. De Lorenzo, AND A. Viru. Hormonal responses to whole-body vibration in men. Eur. J. Appl. Physiol. 81:449-454. 2000.

4. Burke, D., K.E. Hagbarth, L. LÖfstedt, and B.G. Wallin. The responses of human muscle spindle endings to vibration of non-contracting muscles. J. Physiol. 261:673-693. 1976.

5. Burke, D., K.E. Hagbarth, L. Löfstedt, and B.G. Wallin. The responses of human muscle spindle endings to vibration during isometric contraction. J. Physiol. 261:695-711. 1976.

6. Burke, D., AND H.H. Schiller. Discharge pattern of single motor units in the tonic vibration reflex of human triceps surae. J. Neurol. Neurosurg. Psychiatry 39:729-741. 1976.

7. Cardinale, M., ANd C. Bosco. The use of vibration as an exercise intervention. Exerc. Sport Sci. Rev. 31:3-7. 2003.

8. CARDINALE, M., AND J. LIM. Electromyography activity of vastus lateralis muscle during whole-body vibrations of different frequencies. J. Strength Cond. Res. 17:621-624. 2003.

9. De Ruiter, C.J., R.M. VAN DER Linden, M.J.A. VAN DER ZIJDEN, A.P. HOLLANDER, AND A. DE HAAN. Short-term effects of whole-body vibration on maximal voluntary isometric knee extensor force and rate of force rise. Eur. J. Appl. Physiol. 88:472475. 2003.

10. De Ruiter, C.J., S.M. VAN RaAk, J.V. Schilperoort, A.P. Hollander, AND A. DE HAAN. The effects of 11 weeks whole body vibration training on jump height, contractile properties and activation of human knee extensors. Eur. J. Appl. Physiol. 90:595-600. 2003.

11. Delecluse, C., M. Roelants, and S. Verschueren. Strength increase following whole-body vibration compared to resistance training. Med. Sci. Sports Exerc. 35:1033-1041. 2003.

12. Hagbarth, K.E., AND G. EkLund. Tonic vibration reflexes (TVR) in spasticity. Brain Res. 2:201-203. 1966.

13. Issurin, V.B., AND G. Tenenbaum. Acute and residual effects of vibratory stimulation on explosive strength in elite and amateur athletes. J. Sport Sci. 17:177-182. 1999. 
14. LanCE, J.W., D. BuRKe, AND C.J. ANDREws. The reflex effects of muscle vibration. In: New Developments in Electromyography and Clinical Neurophysiology. J.E. Desmedt, ed. Basel: Karger, 1973. pp. 444-462.

15. Martin, B.J., AND H. PARK. Analysis of the tonic vibration reflex: Influence of vibration variables on motor unit synchronization and fatigue. Eur. J. Appl. Physiol. 75:504-511. 1997.

16. Mester, J., P. Spitzenfeil, J. Schwarzer, ANd F. Seifriz. Biological reaction to vibration: Implications for sport. J. Sci. Med. Sport 2:211-226. 1999.

17. NoRdin, M., AND K.E. HAGBARTH. Effects of preceding movements and contractions on the tonic vibration reflex of human finger extensor muscles. Acta Physiol. Scand. 156:435-440. 1996.

18. PARK, H.S., AND B.J. MARTin. Contribution of the tonic vibration reflex to muscle stress and muscle fatigue. Scand. J. Work Environ. Health 19:35-42. 1993.

19. Roelants, M., C. Delecluse, M. Goris, and S. Verschueren. Effects of 24 weeks whole body vibration training on body composition and muscle strength in untrained females. Int. J. Sports Med. 25:1-5. 2004.

20. Roelants, M., C. Delecluse, and S. Verschueren. Whole body vibration training increases knee-extension strength and speed of movement in older women. J. Am. Geriatr. Soc. 52: 901-908. 2004.
21. Rohmert, W., H. Wos, S. Norlander, ANd R. Helbig. Effects of vibration on arm and shoulder muscles in three body postures. Eur. J. Appl. Physiol. Occup. Physiol. 59:243-248. 1989.

22. ROLL, J.P., J.P. VEDEL, AND E. RIBOT. Alteration of proprioceptive messages induced by tendon vibration in man: A microneurographic study. Exp. Brain Res. 76:213-222. 1989.

23. SALE, D.G. Influence of exercise training on motor unit activation. Exerc. Sport Sci. Rev. 15:95-151. 1987.

24. Torvinen, S., P. Kannus, H. Sievänen, T.A.H. Järvinen, M. Pasanen, S. Kontulainen, T.L.N. Järvinen, M. Järvinen, P. OJA, AND I. VUORI. Effect of a vibration exposure on muscular performance and body balance. Randomized cross-over study. Clin. Physiol. Funct. Imaging 22:145-152. 2002.

25. Torvinen, S., P. Kannus, H. Sievänen, T.A.H. Järvinen, M. Pasanen, S. Kontulainen, T. JÄrVInen, T.L.N. JÄrVInEN, M. JÄRVINEN, P. OJA, AND I. VUORI. Effect of four-month vertical whole body vibration on performance and balance. Med. Sci. Sports Exerc. 34:1523-1528. 2002.

\section{Acknowledgments}

We are grateful to Guus van der Meer, Jelte Tempelaars, and Nick De Poot for placing the WBV platform at our disposal (Power Plate). We also thank Dirk Maes and Vincent Weyten for helping with the data acquisition. The cooperation of the subjects is greatly appreciated.

Address correspondence to Sabine M.P. Verschueren, Ph.D., sabine.verschueren@faber.kuleuven.be. 